\title{
薄膜苂光传感
}

刘鸣华

中国科学院化学研究所, 北京 100190

\section{Film-Based Fluorescence Sensing}

\section{Minghua Liu}

Institute of Chemistry, Chinese Academy of Sciences, Beijing 100190, China.

Email: liumh@iccas.ac.cn

Published online: December 16, 2020.

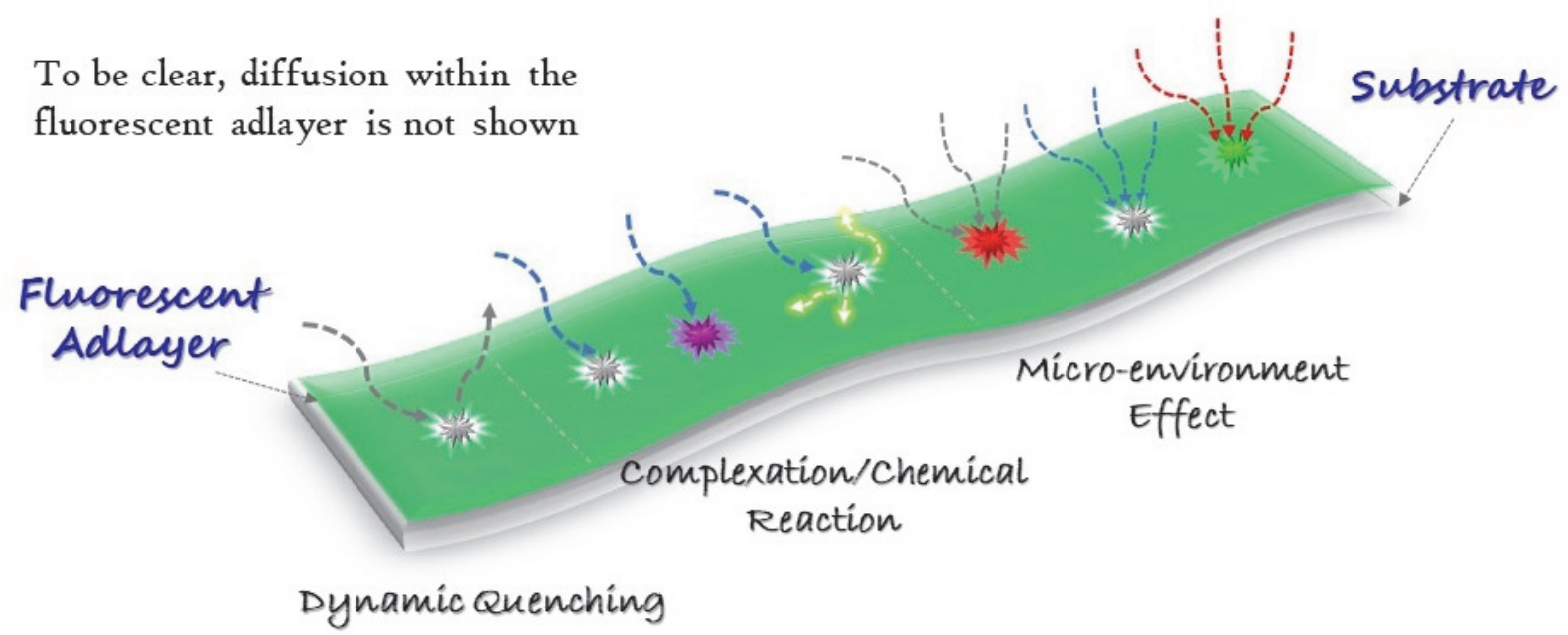

薄膜荧光传感的三种可能机理。

不同于其它探测技术，苂光以激发态实现传 感。因此, 苂光传感具有灵敏度高、可设计性好、 仪器结构相对简单、能耗低、不涉及放射性源、易 于实现便携等优点, 受到了人们的特别关注 ${ }^{1,2}$ 。薄 膜苂光传感是苂光传感的一种重要形式, 是继离 子迁移谱技术之后最具发展潜力的可实现便携的 气相微痕量物质探测技术。敏感薄膜创制是薄膜 荧光技术的核心, 薄膜性能主要取决于决定传感 发生机制的传感单元, 以及影响传感对象分子吸 附、解析和扩散的活性层(adlayer)结构。一般而言, 传感可经由三种机制发生, 一是传感单元与传感 对象分子间的传能, 二是传感单元与传感对象分 子间的特异结合或反应, 三是传感对象分子对传 感单元微环境的扰动。需要指出的是, 无论传感经 由那种机制发生, 传感单元所在活性层的结构都
极大地影响着薄膜的传感性能, 特别是传感响应 动力学。因此, 传感单元结构创新和活性层结构控 制制备对于高性能荧光敏感薄膜的创制具有决定 性意义。

基于上述理念, 陕西师范大学房喻教授团队 提出了综合利用 “苂光单元激发态对微环境的敏 感性”、“活性层微纳结构的毛细效应”, 以及 “吸附、解析、扩散动力学” 设计制备高性能荧光 敏感薄膜的思想, 并首次将传感响应动力学用于 区分检测 ${ }^{3}$ 。在工作中, 他们以高稳定芢二酰亚胺 $(\mathrm{PBI})$ 、碳硼烷芳烃衍生物等为核心结构, 设计制 备了一系列溶解性好, 具有非平面结构特点的苂 光小分子, 以其为传感单元构建了多种富含分子 通道的荧光薄膜, 实现了对爆炸物 (含液体爆炸 物)、毒品、BTEX (苯、甲苯、乙基苯、二甲苯)、 
尼古丁、甲醇等重要化学物质和湿度 ${ }^{3-5}$ 的气相灵 敏可逆探测。以四配位8-差基喹啉硼为基本结构 单元, 设计制备了多种包含非平面结构节点的交 替共聚型荧光聚合物和以其为基础的高性能胃癌 标志物正戊烷气相灵敏可逆探测薄膜。

与美国犹他大学Stang教授合作, 房喻教授团 队通过配位组装构建了多种包含PBI片段, 具有非 平面结构特点的环状、笼状荧光分子, 实现了分子 内PBI片段间激子耦合的调控, 发展了芳胺等有毒 有害化学品的高性能荧光传感薄膜7,8。在对新型 传感单元研究过程中, 房喻教授团队还首次发现 了溶液中芳烃-氢 $(\mathrm{Ar}-\mathrm{H})$ 参与的分子内弱氢键作 用 9 。最近, 房喻教授团队利用动态共价键化学, 在界面限域条件下制备得到了多种自支撑、无缺 陷、结构明确的苂光薄膜, 实现了对假酒的非浸入 式可靠区分检测 10 。这一薄膜制备策略的引入为活 性层结构的控制制备、为传感响应动力学的模型 研究, 以及为柔性薄膜苂光传感研究奠定了坚实 的基础。

上述研究极大地推动了薄膜苂光传感技术 的发展, 彰显了需求牵引下的传感单元结构创新、 活性层结构控制制备, 以及物质迁移研究的重要 意义。

\section{References}

(1) Ding, L.; Fang Y. Chem. Soc. Rev. 2010, 39, 4258. doi: $10.1039 / \mathrm{C} 003028 \mathrm{G}$
(2) Swager, T. M.; Mirica, K. A. Chem. Rev. 2019, 119, 1. doi: 10.1021/acs.chemrev.8b00764

(3) Liu, K.; Shang, C.; Wang, Z.; Qi, Y.; Miao, R.; Liu, K.; Liu, T.; Fang, Y. Nat. Commun. 2018, 9, 1695.

doi: 10.1038/s41467-018-04119-6

(4) Liu, T.; Miao, R.; Peng, H.; Liu, J.; Ding, L.; Fang, Y. Acta Phys. -Chim. Sin. 2020, 36, 1908025. [刘太宏, 苗荣, 彭浩南, 刘静, 丁立平, 房喻. 物理化学学报. 2020,36, 1908025.] doi: 10.3866/PKU.WHXB201908025

(5) Wang, Z.; Wang, G.; Chang, X.; Liu, K.; Qi, Y.; Shang, C.; Huang, R.; Liu, T.; Fang, Y. Adv. Funct. Mater. 2019, 25, 1905295. doi: 10.1002/adfm.201905295

(6) Qi, Y.; Xu, W.; Kang, R.; Ding, N.; Wang, Y.; He, G.; Fang, Y. Chem. Sci. 2018, 9, 1892. doi: 10.1039/C7SC05243J

(7) Chang, X.; Zhou, Z.; Shang, C.; Wang, G.; Wang, Z.; Qi, Y.; Li, Z.-Y.; Wang, H.; Cao, L.; Li, X.; Fang, Y.; Stang, P. J. J. Am. Chem. Soc. 2019, 141, 1757. doi: 10.1021/jacs.8b12749

(8) Chang, X.; Lin, S.; Wang, G.; Shang, C.; Wang, Z.; Liu, K.; Fang, Y.; Stang, P. J. J. Am. Chem. Soc. 2020, 142, 37, 15950. doi: $10.1021 /$ jacs.0c06623

(9) Shang, C.; Wang, G.; Liu, K.; Jiang, Q.; Liu, F.; Chou, P.-T.; Fang, Y. Angew. Chem. Int. Ed. 2020, 59, 8579. doi: 10.1002/anie. 201914070

(10) Han, T.; Yang, J.; Miao, R.; Liu, K.; Li, J.; Wang, D.; Liu, T.; Fang, Y. Adv. Mater. Technol. 2020, 2000933.

doi: 10.1002/admt.202000933 